\title{
Line of Sight: Improving Supply Chain Visibility
}

\section{Steve Cottrell}

Maetrics, 8888 Keystone Crossing, Suite 1550, Indianapolis, IN 46240, USA

There can be few sectors where the quality of purchased materials and of outsourced activities are more important than in pharmaceuticals. However, while pharmaceutical companies are ultimately in charge of ensuring that both consistently meet the necessary standards, the complexity of multi-tiered supply chains, as well as inadequate - or even a lack of - IT systems, limited experience, and resource constraints can all combine to make this a difficult task.

Globally, research shows that full supply chain visibility rose to third most important strategic priority for professionals with a direct link to the process in their organisation in 2017, up from sixth in 2015, yet only $6 \%$ of firms say they have achieved this level of scrutiny [1]. The pharmaceutical supply chain has certainly become more complicated in recent years due to the entrance of vendors from countries that previously played a limited role, such as China and India where there is numerous excipient, chemical, API and CMO suppliers.

Another factor clouding supply chain visibility is the knock-on effect of mergers and acquisitions. While both these and new vendors can also bring many positives, any lack of visibility in the supply chain introduces risks. In many cases the paring back of manufacturing operations often made by the acquiring companies, limits the ability of personnel to perform supply chain gap analyses, supplier assessments, and to manage any quality issues that may arise. This can in turn result in any number of issues from the inability to ensure compliance, to difficulties in fully understanding demand and capacity, and to assessing and reacting to any disruptions, all of which will affect quality and performance.

Operations can also be delayed for a number of reasons and it is imperative that the root cause can be traced in order for problems to be solved. Materials may arrive that do not conform to specifications or lack a Certificate of Analysis or other required certifications, for example. Other issues could be that the materials do not perform during the manufacturing process because of supplier error, poor shipping temperature controls, or data integrity issues at the supplier that internal auditors were not adequately trained to spot. Poorly constructed Quality Agreements with suppliers also cause issues, for example by not including any legal requirement for a supplier to inform their client of quality issues encountered during manufacture of the API.

These can all become major issues without supply chain visibility, and in fact, lack of coordination, inventory management, order management, and temperature control are all in the top 10 global health pharmaceutical supply chain challenges according to one 2015 report [2].

Following the supplier quality outsourcing model for example has been shown to enable as much as $48 \%$ cost savings per year, achieved by managing costs per audit versus full-time equivalent (FTE) resources, so maintaining a higher level of compliance and inspection readiness while allowing internal resources to focus on other business needs.

There are business risks which are associated with supplier quality; while pharmaceutical companies are ultimately responsible to assure the control and review of outsourced activities and the quality of purchased materials, many in fact struggle with this, due to the complexity of multi-tiered supply chains, the lack of or inadequacy of IT systems, limited experience and resource constraints. Some of the key business risks associated with supplier quality are: supply reliability, sustainability, contract adherence, supply chain security, fraud prevention and confidentiality.

While a lack of visibility can result in any one or more of these problems arising, there are many processes that, through leveraging technology and real-time data, will serve to dramatically reduce them once implemented. The strategic benefits of an improved supplier quality evaluation include both a higher level of compliance and inspection readiness, an increased understanding of demand and capacity, ability to assess and react to disruptions and indirect cost savings which are related to increased Quality of Supplier Output. Ultimately improving supply chain visibility will result in increased quality and performance as companies will minimise risk through greater understanding and being generally better equipped and prepared to react, as and when is needed.

Key steps to improving supply chain visibility:

- Implement processes to control and review outsourced activities and quality of purchased materials. These should incorporate quality risk management, regular monitoring and review of suppliers.

- Leverage real time data: only by using this will you gain an accurate, up-to-date view of the supply chain.

- Keep detailed records of the quality of the lots received in addition to the on-time receipt of materials.

- Ensure you have on-site audits in place, especially the qualification and re-qualifications audit, and for-cause investigations.

- Regularly monitor and review suppliers, using periodic scorecard assessments, interim re-audits, SCAR issuance, and investigative reviews to maintain supply chain quality.

- Monitor geographic and geopolitical environments round the clock to head off potential supply chain interruptions.

- Assess your supplier and quality agreements: well-planned, wellwritten, and successfully executed agreements are essential to control the process and quality of the final product.

The increasing complexity of the pharmaceutical supply chain means full visibility is absolutely critical both for preventing problems

*Corresponding author: Steve Cottrell, President, Maetrics, 8888 Keystone Crossing, Suite 1550, Indianapolis, IN 46240, USA, Tel: 317-706-1493; E-mail: scottrell@maetrics.com

Received February 09, 2018; Accepted February 19, 2018; Published February 27,2018

Citation: Cottrell S (2018) Line of Sight: Improving Supply Chain Visibility. Pharmaceut Reg Affairs 7:1. doi: 10.4172/2167-7689.1000200

Copyright: (c) 2018 Cottrell S. This is an open-access article distributed under the terms of the Creative Commons Attribution License, which permits unrestricted use, distribution, and reproduction in any medium, provided the original author and source are credited. 
from occurring and for efficiently solving them when they do. Certainly, until a company has this clear view of every stage of the chain, ensuring standards are met will prove an ever-growing challenge. Partnering with experienced consultants can help harness these processes and ensure the supply chain is kept under close check.

\section{References}

1. GEODIS (2017) GEODIS Unveils its 2017 Supply Chain Worldwide Survey. Bruno DELFILS, Levallois-Perret, France.

2. Beswick L (2015) Top 10 Challenges Facing Global Pharmaceutical Supply Chains. GT Nexus. 